\title{
Safety and efficacy of cement augmentation with fenestrated pedicle screws for tumor-related spinal instability
}

\author{
Elie Massaad, MD, Myron Rolle, MD, Muhamed Hadzipasic, MD, PhD, Ali Kiapour, PhD, \\ Ganesh M. Shankar, MD, PhD, and John H. Shin, MD
}

Department of Neurosurgery, Massachusetts General Hospital, Harvard Medical School, Boston, Massachusetts

\begin{abstract}
OBJECTIVE Achieving rigid spinal fixation can be challenging in patients with cancer-related instability, as factors such as osteopenia, radiation, and immunosuppression adversely affect bone quality. Augmenting pedicle screws with cement is a strategy to overcome construct failure. This study aimed to assess the safety and efficacy of cement augmentation with fenestrated pedicle screws in patients undergoing posterior, open thoracolumbar surgery for spinal metastases.
\end{abstract}

METHODS A retrospective review was performed for patients who underwent surgery for cancer-related spine instability from 2016 to 2019 at the Massachusetts General Hospital. Patient demographics, surgical details, radiographic characteristics, patterns of cement extravasation, complications, and prospectively collected Patient-Reported Outcomes Measurement Information System Pain Interference and Pain Intensity scores were analyzed using descriptive statistics. Logistic regression was performed to determine factors associated with cement extravasation.

RESULTS Sixty-nine patients underwent open posterior surgery with a total of 502 cement-augmented screws (mean 7.8 screws per construct). The median follow-up period for those who survived past 90 days was 25.3 months (IQR 10.8-34.6 months). Thirteen patients (18.8\%) either died within 90 days or were lost to follow-up. Postoperative CT was performed to assess the instrumentation and patterns of cement extravasation. There was no screw loosening, pullout, or failure. The rate of cement extravasation was $28.9 \%$ (145/502), most commonly through the segmental veins (77/145, $53.1 \%)$. Screws breaching the lateral border of the pedicle but with fenestrations within the vertebral body were associated with a higher risk of leakage through the segmental veins compared with screws without any breach (OR 8.77, 95\% $\mathrm{Cl} 2.84-29.79 ; p<0.001)$. Cement extravasation did not cause symptoms except in 1 patient who developed a symptomatic thoracic radiculopathy requiring decompression. There was 1 case of asymptomatic pulmonary cement embolism. Patients experienced significant pain improvement at the 3-month follow-up, with decreases in Pain Interference (mean change 15.8, 95\% Cl 14.5-17.1; $p<0.001$ ) and Pain Intensity (mean change 28.5, 95\% Cl 26.7-30.4; $p<0.001$ ).

CONCLUSIONS Cement augmentation through fenestrated pedicle screws is a safe and effective option for spine stabilization in the cancer population. The risk of clinically significant adverse events from cement extravasation is very low. https://thejns.org/doi/abs/10.3171/2021.2.FOCUS201121

KEYWORDS cement augmentation; complications; fenestrated screw; PROMIS; spine metastasis; spine surgery

$\mathrm{P}$ ATIENTS experiencing pain related to spinal column metastases often undergo surgery to alleviate mechanical pain that is not palliated effectively with interventional, radiation, or medical therapies. ${ }^{1-3}$ Considering the degree to which pathologic fracture, spinal cord compression, and spinal instability can compromise the quality of life for these patients, it is critical to maximize the biomechanical properties of fixation when sta- bilization is required. ${ }^{4,5}$ Hardware failure in this context has been reported to range from $1.9 \%$ to $13.8 \%$ in contemporary series. ${ }^{6-8}$ Surgical complications and need for revision surgery can have deleterious effects on recovery, survival, and timely initiation of radiation and oncological therapies. ${ }^{9-12}$ Therefore, improving surgical strategies to reduce implant-related failures is paramount, as achieving arthrodesis in a bone marrow environment adversely af-

ABBREVIATIONS AIS = American Spinal Injury Association Impairment Scale; ASA = American Society of Anesthesiologists Physical Status Classification; ECOG = Eastern Cooperative Oncology Group; ESCC = epidural spinal cord compression; NOMS = neurological, oncological, mechanical, and systemic; PMMA = polymethylmethacrylate; PROMIS = Patient-Reported Outcomes Measurement Information System; SINS = Spine Instability Neoplastic Score.

SUBMITTED December 31, 2020. ACCEPTED February 16, 2021

INCLUDE WHEN CITING DOI: 10.3171/2021.2.FOCUS201121. 


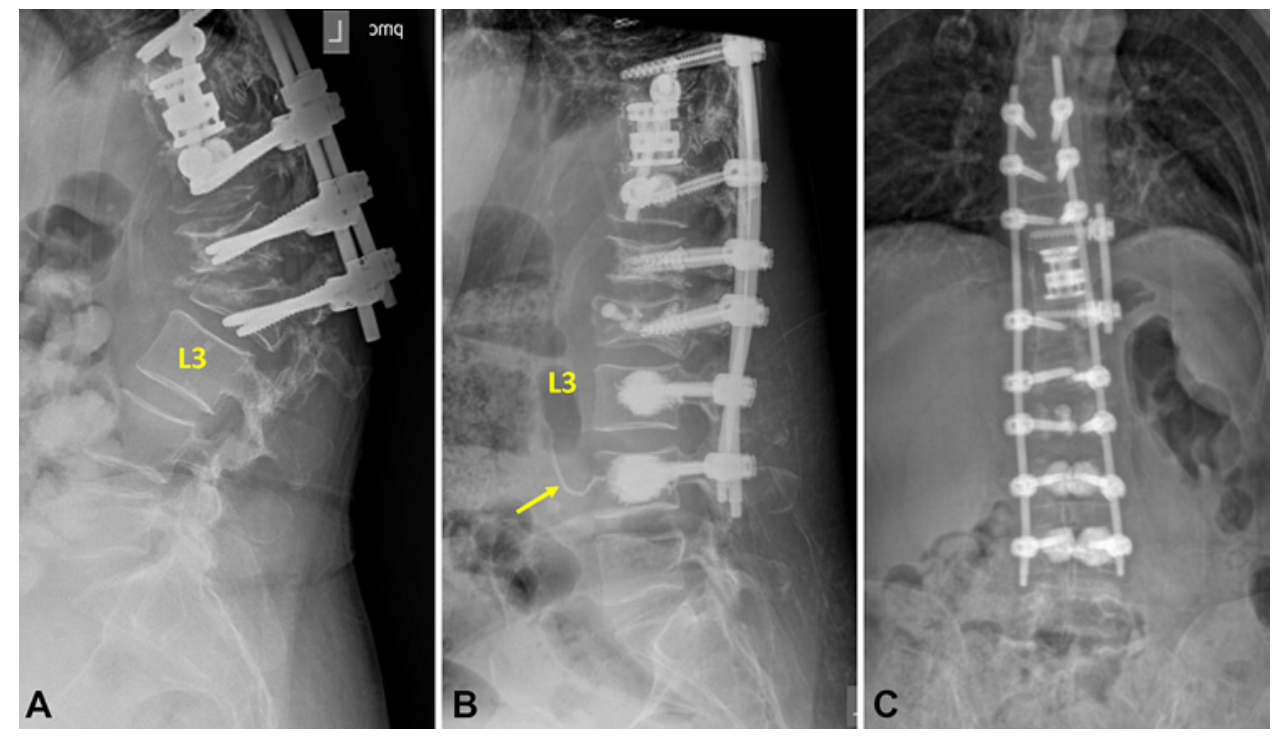

FIG. 1. Case illustration. A 67-year-old woman with metastatic breast cancer underwent combined anterior and posterior surgery, T8-L2, for T11 pathologic fracture and Bilsky grade 3 ESCC in 2009, followed by conventional fractionated radiation therapy at another institution. The patient improved from AIS grade C to $E$ and presented in 2018 with multilevel disease progression, severe mechanical pain, a SINS of 16, and kyphosis from a new pathologic fracture at $L 2$. The patient was not able to stand upright due to severe pain. A: Preoperative lateral standing lumbar radiograph showing compression deformities of $L 1$ and $L 2$, thoracolumbar kyphosis, and distal instrumentation failure with pullout of the $L 2$ screws. The patient underwent posterior revision, bilateral posterior column osteotomies at L1-2, and placement of fenestrated screws with cement augmentation at L1, L2, L3, and L4. B: Lateral standing lumbar radiograph obtained 6 months postoperatively, showing correction of kyphosis and preserved restoration of alignment. The yellow arrow points to cement extravasation into a segmental vein at L4. Limited cement was placed in L1 and L2 due to the fracture morphology and extent of collapse of the vertebral bodies. C: Anteroposterior standing lumbar radiograph obtained 6 months postoperatively.

fected by factors such as osteoporosis, radiation, immunosuppression, and malnutrition is limited. ${ }^{6,13-15}$

To overcome the anticipated poor bone quality in these patients, polymethylmethacrylate (PMMA) bone cement has been used as an adjunct when placing pedicle screws. ${ }^{16}$ With advances in instrumentation technology and cement delivery systems, fenestrated screws permit injection of cement into the vertebral body through distal openings. Before fenestrated screws were available, cement would typically have been injected through cannulas directly into the vertebral body prior to screw placement. ${ }^{17}$ Biomechanical and clinical studies have reported using such techniques for osteoporotic conditions, yet only one report has specifically studied this in the metastatic cancer population. ${ }^{18-20}$

To our knowledge, the current study is the largest series examining the clinical, surgical, and radiological factors associated with safety, outcomes, and pain palliation using this technology in open metastatic spine tumor surgery.

\section{Methods}

This retrospective study included medical record data from all patients older than 18 years of age treated surgically for spinal metastases with fenestrated screws between 2016 and 2019 at the Massachusetts General Hospital. Included patients underwent open surgery for decompression and stabilization of the spine for diagnoses of epidural spinal cord compression (ESCC), vertebral pathologic fracture, or mechanical instability from cancer.
Commercially available, FDA-approved fenestrated screw systems became available at our institution at the end of 2016 (DePuy Synthes and Medtronic). Institutional review board approval was obtained and informed consent for retrospective analysis of de-identified data was waived.

\section{Selection Criteria}

Patients were selected for surgery based on a decisionmaking algorithm incorporating the neurological, oncological, mechanical, and systemic (NOMS) framework; Spine Instability Neoplastic Score (SINS); and multidisciplinary assessment of frailty and anticipated survival. ${ }^{3,5}$ ${ }_{21,22}$ Only patients diagnosed with metastatic spinal tumors were included in this study.

Posterior thoracolumbar decompression and stabilization with fenestrated pedicle screws was performed through an open midline approach for the following scenarios: ESCC; mechanical oncological instability; and deformity, fracture, or kyphosis following radiation therapy (Fig. 1). All patients who underwent surgery had either intractable mechanical pain or symptomatic compression of the spinal cord or nerve roots. Cases involving percutaneous or minimal-access techniques were excluded. Patient-reported pain scores were recorded prospectively at baseline preoperatively and at the 3-month follow-up using Patient-Reported Outcomes Measurement Information System (PROMIS) Short Form v1.1 Pain Interference 6a and Short Form v2.0 Pain Intensity 3a. ${ }^{23,24}$ Raw scores from the surveys were converted to a $t$-score with a mean 
of 50 and a standard deviation of 10 . Higher scores indicate more of the concept being measured (i.e., pain).

Fenestrated screws with cement augmentation were placed at each vertebral body level in consecutive cases where CT and/or MRI demonstrated osteopenic, lytic changes consistent with bony metastases. Blastic and mixed lytic-blastic levels were not instrumented with fenestrated screws.

\section{Pedicle Screw Insertion and Cement Injection Technique}

All surgeries were performed under general anesthesia with patients in the prone position on a Jackson table. After exposure, pedicles from $\mathrm{T} 1$ to $\mathrm{S} 1$ were cannulated with a straight gear shift instrument using anatomical landmarks and freehand techniques prior to decompression. Pedicle markers were placed into each pedicle, and the trajectories into the vertebral bodies were verified using intraoperative fluoroscopy. If a pedicle marker was not positioned satisfactorily, the trajectory was revised, and a standard pedicle screw was placed as no attempt would be made to augment at that site for concern of cement extravasation. The accuracy of pedicle screw placement was verified using fluoroscopy in all cases. In most cases, separation surgery was performed. ${ }^{25}$ Transpedicular corpectomy with anterior column reconstruction was performed in cases of hypervascular tumors with significant vertebral body bleeding, locally recurrent disease after previous intralesional decompression surgery, or kyphotic deformity.

Cement was mixed after the screw position was verified and injected one screw at a time under fluoroscopic guidance after the desired consistency was achieved. A volume of $1.5 \mathrm{~mL}$ of cement was used in accordance with manufacturer specifications through each pedicle unless there was evidence of extravasation, at which time the fill was terminated.

\section{Radiological Evaluation}

Postoperative CT was performed to assess the placement of instrumentation and pattern of cement fill and extravasation after each case. The position of each screw was recorded using the Heary classification. ${ }^{26}$ In this system, well-positioned screws that did not breach the borders of the pedicle were classified as grade 1; screws breaching the pedicle laterally but with the tip inside the vertebral body were grade 2; screws breaching anteriorly were grade 3; screws breaching medially or inferiorly were grade 4; and screws in the spinal canal or compromising neurovascular structures were grade 5. A screw was considered breached in any direction irrespective of the extent of extracortical violation if the breach distance was greater than $0 \mathrm{~mm} .{ }^{27}$

The primary pattern of cement leakage was recorded using the Yeom classification: type $\mathrm{S}$ (leakage via the vertebral segmental veins), type $\mathrm{C}$ (leakage via a cortical defect), and type B (leakage into the basivertebral vein and into the spinal canal). ${ }^{28}$ Any question regarding the classification of instrumentation or the primary pattern of cement extravasation was reconciled by discussion. Instrumentation-related complications, including screw pullout, lucency, fracture, rod fracture, and development of deformity or malalignment, were evaluated using CT during follow-up.

\section{Statistical Analysis}

Descriptive analyses of patient demographics, surgery, radiological outcomes, medical and surgical complications, and patient-reported pain outcomes were performed. Wilcoxon rank-sum tests were employed to compare the changes in PROMIS Pain Intensity and Pain Interference before and after surgery. Logistic regression was performed to evaluate the association between the pattern of cement leakage and the position of the pedicle screws. These associations were reported using estimates, 95\% confidence intervals, and 2 -tailed $\mathrm{p}$ values. $\mathrm{P}$ values were considered significant if they were $<0.05$. All analyses were performed using R version 3.6.1 (The R Foundation) and RStudio version 1.2.5001.

\section{Results}

Sixty-nine patients were included in the analysis. Cement augmentation was performed through 502 fenestrated pedicle screws with a mean of $7.8 \pm 2.4$ screws implanted per case. The most common construct involved placing fenestrated instrumentation 2 levels above and 2 levels below the pathological level of compression or kyphosis. Separation surgery was performed in 56 patients (81.2\%), posterior transpedicular corpectomy with anterior column reconstruction surgery in 10 patients (14.5\%), and stabilization surgery for tumor-related instability in 3 patients $(4.3 \%)$. The mean age at the time of surgery was $64.7 \pm 10.3$ years (median age [range] 67 [30-84] years), and 38 patients $(55.1 \%)$ were male. The median follow-up period for those who survived past 90 days $(\mathrm{n}=56,81.2 \%$ ) was 25.3 months (IQR 10.8-34.6 months). Most patients (78.3\%) were classified as American Society of Anesthesiologists Physical Status Classification (ASA) class II. The most common tumors were lung (18.8\%), renal (14.5\%), and breast (10.1\%).

Most patients $(\mathrm{n}=52,75.4 \%)$ had a pathologic fracture at the index level. Preoperative assessments revealed that 51 patients $(73.9 \%)$ had intermediate instability (SINS 7-12) and 18 (26.1\%) had highly unstable spines (SINS 13-18). High-grade epidural compression (ESCC Bilsky grade 2-3) was present in 53 cases (76.8\%). Assessment of performance status using the Eastern Cooperative Oncology Group (ECOG) scale showed that 14 patients (20.3\%) were fully functional (ECOG grade 0 ). Most patients were ECOG grade $1(n=32,46.4 \%)$. Prior to surgery, $40.5 \%$ of patients had some degree of motor weakness, indicated on the American Spinal Injury Association (ASIA) Impairment Scale (AIS) as grade B-D. Potential factors affecting bone quality were recorded (Table 1). Fourteen patients $(20.3 \%)$ had a known history of osteoporosis, and 28 (40.6\%) had diffuse osteopenia by imaging reports. Prior to surgery, 21 patients (30.4\%) had received radiation to the spine and $45(65.2 \%)$ were actively on systemic treatment.

\section{Radiographic Evaluation}

The distribution of instrumented spinal levels between 
TABLE 1. Patient and tumor characteristics

\begin{tabular}{|c|c|}
\hline & Value \\
\hline No. of patients & 69 \\
\hline Mean age \pm SD, yrs & $64.7 \pm 10.3$ \\
\hline \multicolumn{2}{|l|}{ Sex } \\
\hline Male & $38(55.1)$ \\
\hline Female & $31(44.9)$ \\
\hline Mean $\mathrm{BMI} \pm \mathrm{SD}, \mathrm{kg} / \mathrm{m}^{2}$ & $27.5 \pm 6.3$ \\
\hline \multicolumn{2}{|l|}{ ASA class } \\
\hline I & $12(17.4)$ \\
\hline II & $54(78.3)$ \\
\hline III & $3(4.3)$ \\
\hline \multicolumn{2}{|c|}{ Preop ECOG functional status } \\
\hline 0 & $14(20.3)$ \\
\hline 1 & $32(46.4)$ \\
\hline 2 & $12(17.4)$ \\
\hline 3 & $10(14.5)$ \\
\hline Missing & $1(1.5)$ \\
\hline \multicolumn{2}{|l|}{ Preop AIS grade } \\
\hline A & $0(0)$ \\
\hline B & $3(4.3)$ \\
\hline C & $1(1.4)$ \\
\hline D & $24(34.8)$ \\
\hline$E$ & $41(59.4)$ \\
\hline \multicolumn{2}{|l|}{ Tumor } \\
\hline Lung & $13(18.8)$ \\
\hline Prostate & $10(14.5)$ \\
\hline Renal & $10(14.5)$ \\
\hline Breast & $7(10.1)$ \\
\hline Colon & $4(5.8)$ \\
\hline Melanoma & $4(5.8)$ \\
\hline Multiple myeloma & $4(5.8)$ \\
\hline Other & $17(24.6)$ \\
\hline \multicolumn{2}{|l|}{ SINS } \\
\hline Intermediate, 7-12 & $51(73.9)$ \\
\hline Unstable, 13-18 & $18(26.1)$ \\
\hline \multicolumn{2}{|l|}{ Bilsky ESCC grade } \\
\hline Low-grade, 0 or 1 & $16(23.2)$ \\
\hline High-grade, 2 or 3 & $53(76.8)$ \\
\hline Pathologic fracture & $52(75.4)$ \\
\hline Previous chemotherapy & $45(65.2)$ \\
\hline Previous radiation therapy & $21(30.4)$ \\
\hline Smoking & $38(55.1)$ \\
\hline Steroid use $>7$ days & $17(24.6)$ \\
\hline Osteopenia & $28(40.6)$ \\
\hline Osteoporosis & $14(20.3)$ \\
\hline
\end{tabular}

Values represent the number of patients (\%) unless stated otherwise.
T1 and S1 is shown in Fig. 2. Most fenestrated pedicle screws $(441 / 502,87.8 \%)$ were placed within the pedicle and the vertebral body (Heary grade 1). Cement extravasation was observed among all screw placement classifications, including Heary grade 1 (107/441, 24.2\%). When extravasation occurred with grade 1 screws (107 total), the most common pattern of spread was through the segmental veins of the vertebral bodies (Yeom type S; 68/107, $63.6 \%$ ). Summary characteristics of screw position and distribution of cement leakage are shown in Fig. 3.

Screws breaching the lateral border of the pedicle but having their tip inside the vertebral body (Heary grade 2) had a higher risk of leakage through the segmental veins (Yeom type $\mathrm{S}$ ) compared with well-positioned screws (OR 8.77, 95\% CI 2.84-29.79; $\mathrm{p}<0.001)$. Screws with the tip violating the anterior vertebral body cortex (Heary grade 3 ) were more likely to have cement leakage through the cortical wall (Yeon type C) compared with screws inserted with no violation of the pedicle or vertebral body (OR 85.01, 95\% CI 29.48-287.86; $p<0.001$ ). Screws breaching the medial or inferior pedicle borders (Heary grade 4) were significantly associated with cement leakage through the basivertebral vein and into the spinal canal (Yeom type B) (OR 14.5, 95\% CI 6.05-34.77; $\mathrm{p}<0.001$ ). Representative CT patterns of cement extravasation are illustrated in Fig. 4.

\section{Patient-Reported Pain Outcomes}

We performed an analysis comparing the prospectively obtained PROMIS Short Form v1.1 Pain Interference 6a and Short Form v2.0 Pain Intensity 3a scores prior to surgery and at 3 months postoperatively (Fig. 5). Of 69 patients, 9 died within 90 days of surgery and 4 were lost to follow-up. Pre- and postoperative pain scores were available for 52 patients. Prior to undergoing surgery, patients scored a mean of $68.2 \pm 2.2$ points on PROMIS Pain Interference and $76.9 \pm 4.7$ points on Pain Intensity. This corresponds to an average of 18.2 and 26.9 points above the normative population (mean $50 \pm 10$ points) in Pain Interference and Pain Intensity, respectively. This demonstrates the severe range of preoperative pain. Patients experienced significant postoperative improvements in both Pain Interference and Pain Intensity. Three months after surgery, the mean improvement in Pain Intensity was 28.5 points (95\% CI 26.7-30.4; p < 0.001) and 15.8 (95\% CI $14.5-17.1 ; p<0.001)$ for Pain Interference. The average postoperative Pain Intensity score was 1.6 points below the normative population $(48.4 \pm 5.4$ points), while the average Pain Interference score was 2.4 points above the normative population $(52.4 \pm 4.6$ points).

\section{Complications}

Intraoperative and postoperative complications are listed in Table 2. Hemodynamic instability as a result of high-volume blood loss during vertebrectomy occurred in 2 patients $(2.9 \%)$ and was not related to screw placement or cement augmentation. No intraoperative adverse events were related to screw insertion or cement augmentation. The 30-day overall complication rate was $34.8 \%$. The most common 30 -day complications were urinary tract 
Massaad et al.

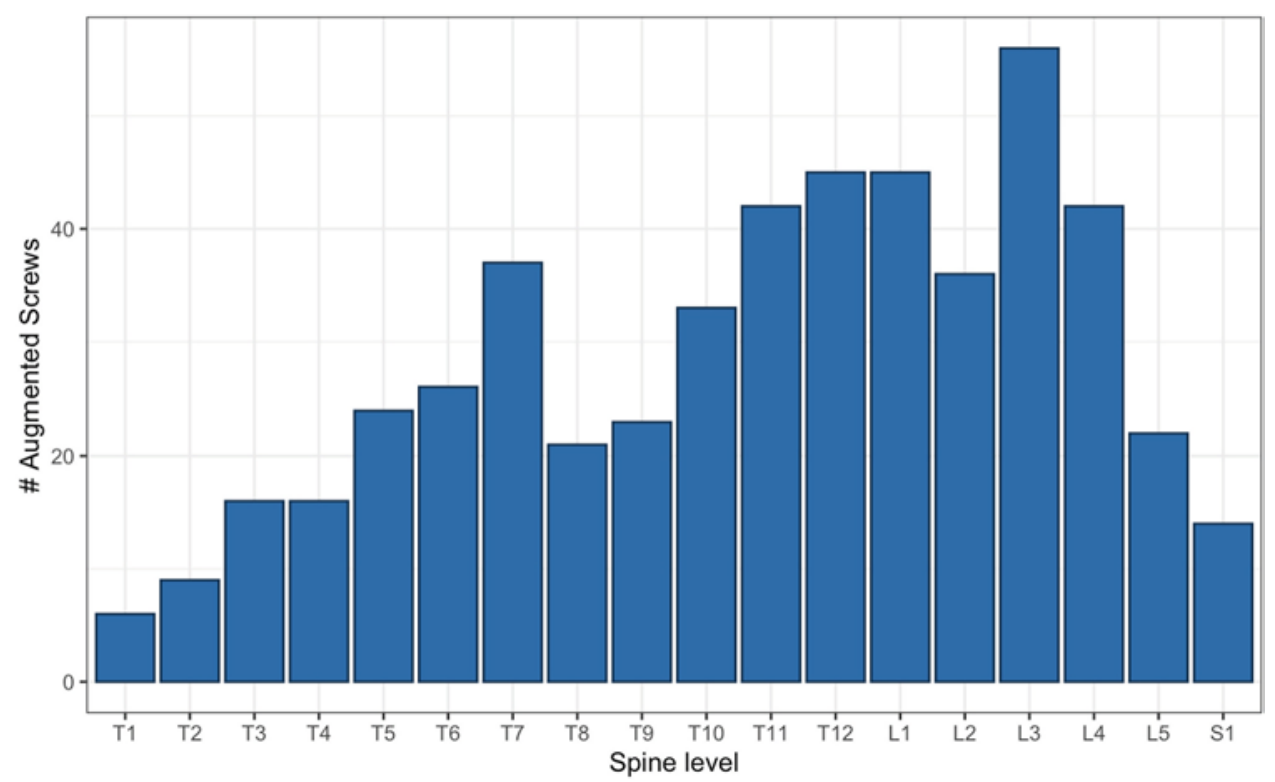

FIG. 2. Bar graph showing the distribution of implanted fenestrated screws from $\mathrm{T} 1$ to S1.

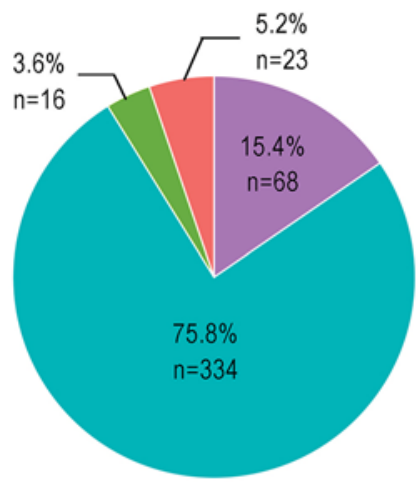

Heary Grade $1(n=441)$

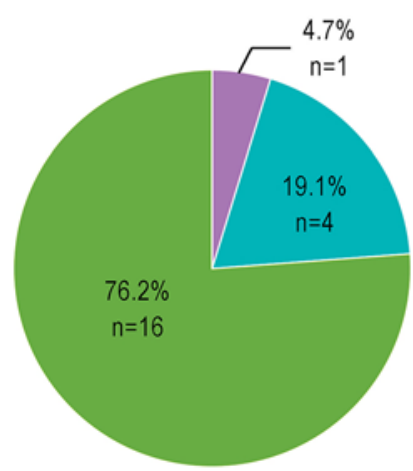

Heary Grade $3(n=21)$

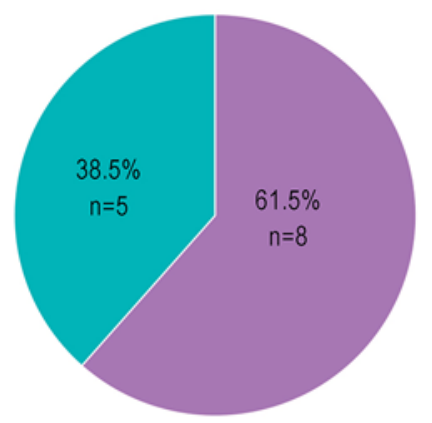

Heary Grade $2(n=13)$

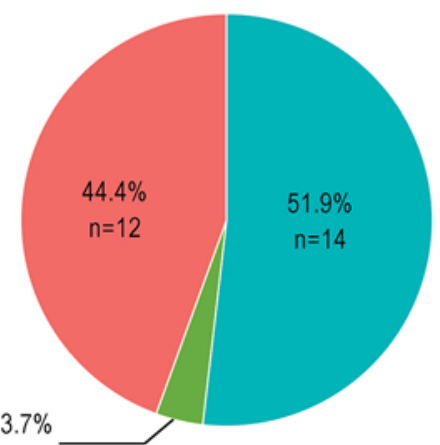

$n=1 \quad$ Heary Grade $4(n=27)$

\section{Yeom et al. Cement Leakage Classification}

Type $\mathrm{B}=$ leakage via the basivertebral vein into the spinal canal

Type $\mathrm{C}=$ leakage through a cortical wall defect

Type $S=$ leakage via the segmental vein of the vertebral body

No cement extravasation or leakage

FIG. 3. Pie charts representing the types of cement leakage and position of the pedicle screws according to the Yeom and Heary classifications, respectively. Heary grade 1: well-positioned screws without breach. Heary grade 2: screws that breach the pedicle laterally but with the tip inside the vertebral body. Heary grade 3: screws that breach the cortex anteriorly. Heary grade 4: screws that breach the pedicle medially or inferiorly. The number of screws and their associated percentages are provided. 
Massaad et al.
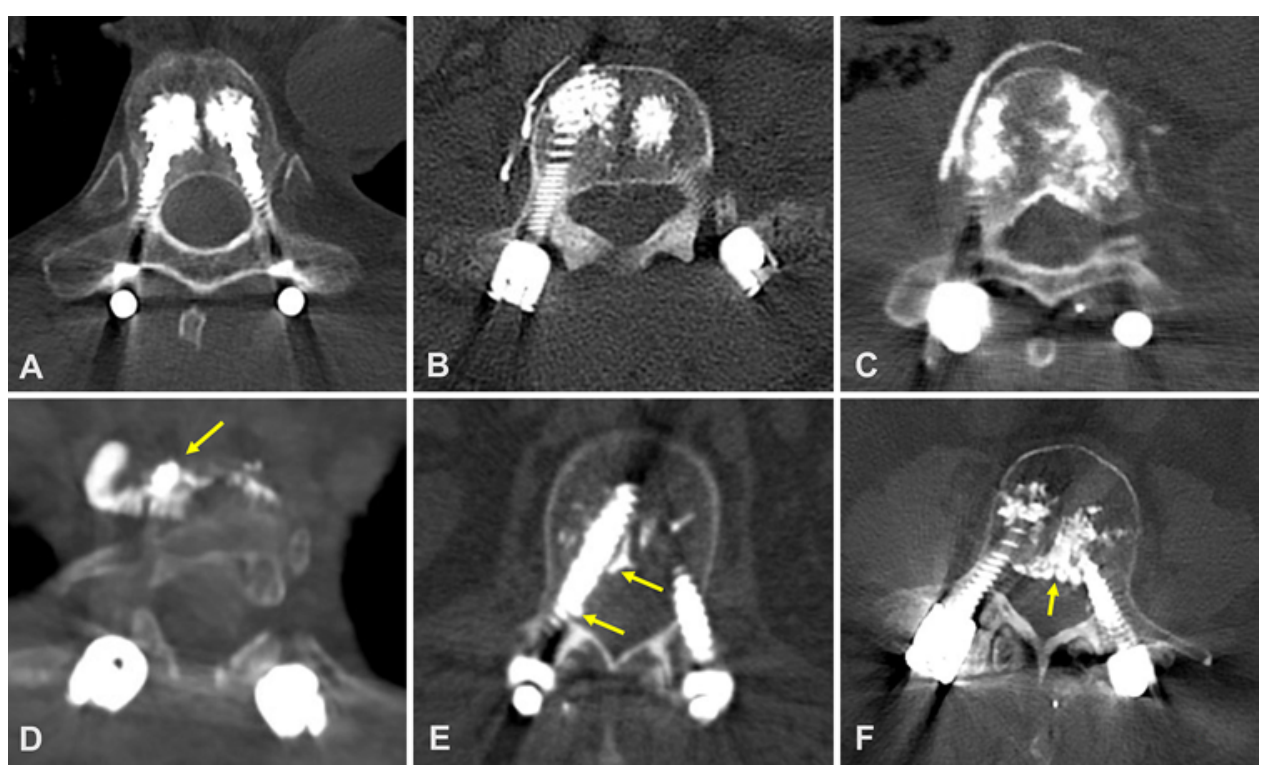

FIG. 4. Representative axial CT images of cement extravasation patterns. A: Well-positioned screws without pedicle breach (Heary grade 1) and no cement extravasation. B: Well-positioned screws (Heary grade 1) with cement extravasation into the right segmental vein (Yeom type S). C: Right lateral screw (in-out-in) with its distal tip in the vertebral body (Heary grade 2) with cement extravasation into the segmental veins (Yeom type S). D: Right screw with its tip (arrow) beyond the anterior cortex (Heary grade 3) and cement beyond the cortex (Yeom type C). E: Right medial screw (Heary grade 4) with cement in the basivertebral vein, canal, and tracking along the medial right pedicle screw (Yeom type B) (arrows). F: Left medial screw (Heary grade 4) with cement in the basivertebral vein and in the canal (Yeom type B) (arrow).

infections ( $\mathrm{n}=5,7.2 \%)$, deep venous thrombosis and pulmonary embolism $(n=3,4.3 \%)$, delirium $(n=2,2.9 \%)$, and wound healing issues requiring surgical site washout and debridement $(\mathrm{n}=5,7.24 \%)$. In 1 patient $(1.45 \%)$, cement extravasation ventral to the T4 nerve root on the medial side of the pedicle was symptomatic, manifesting in severe postoperative chest wall pain that required exploration, decompression, and removal of the extravasated cement.
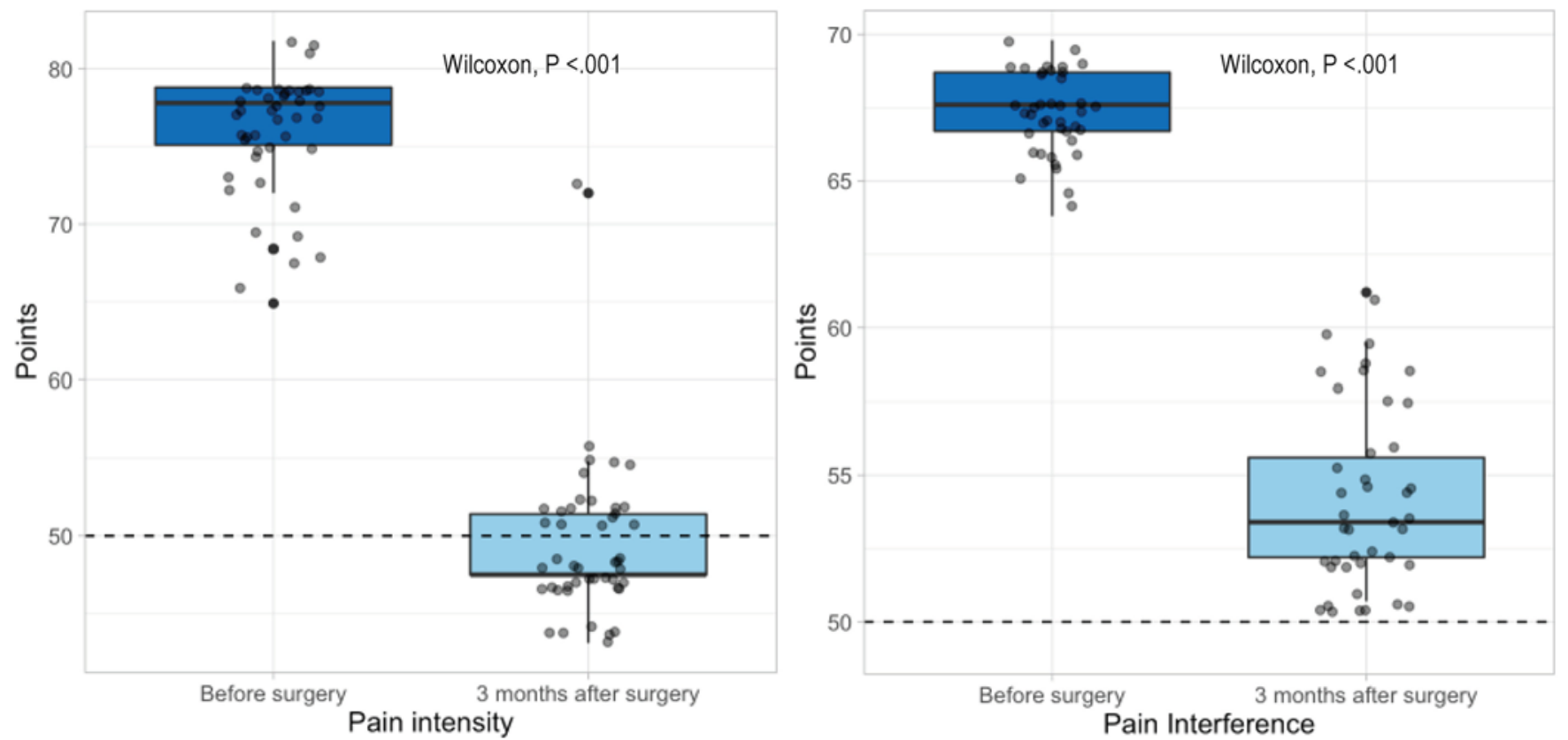

FIG. 5. Boxplots demonstrating significant improvement in PROMIS Pain Intensity (left) and Pain Interference (right) after spine surgery (Wilcoxon test $p<0.001$ ). The distributions are shown as boxplots where the central line is the median concentration, and the edges of the box are the 25 th and 75 th percentiles. The horizontal dashed line represents the average value of the PROMIS domain in the normative population (mean $50 \pm 10$ points). 
TABLE 2. Surgical details

\begin{tabular}{|c|c|}
\hline & Value \\
\hline \multicolumn{2}{|l|}{ Surgery characteristics } \\
\hline Separation surgery & $56(81.2)$ \\
\hline $\begin{array}{l}\text { Transpedicular partial corpectomy w/ anterior } \\
\text { column reconstruction }\end{array}$ & $10(14.5)$ \\
\hline Stabilization alone & $3(4.3)$ \\
\hline $\begin{array}{l}\text { Mean no. of augmented fenestrated pedicle } \\
\text { screws/case } \pm \text { SD }\end{array}$ & $7.8 \pm 2.4$ \\
\hline Mean estimated blood loss, $\mathrm{mL}$ (range) & $300(100-5200)$ \\
\hline Intraop complication/hemodynamic instability & $2(2.9)$ \\
\hline 30-day complications & $24(34.8)$ \\
\hline Wound infection/dehiscence & 5 \\
\hline Urinary tract infection & 5 \\
\hline Deep venous thrombosis/pulmonary embolism & 3 \\
\hline Delirium & 2 \\
\hline Fever of unknown origin & 2 \\
\hline Acute renal failure & 1 \\
\hline Atrial fibrillation & 1 \\
\hline lleus & 1 \\
\hline Pleural effusion & 1 \\
\hline Pneumonia & 1 \\
\hline Pulmonary cement embolism & 1 \\
\hline Sepsis & 1 \\
\hline Reoperation & $9(13)$ \\
\hline Wound revision (w/in 30 days of op) & 5 \\
\hline Disease progression (>90 days postop) & 2 \\
\hline Instrumentation removal (>90 days postop) & 1 \\
\hline $\begin{array}{l}\text { Cement extravasation causing radiculopathy } \\
\text { (w/in } 30 \text { days of op) }\end{array}$ & 1 \\
\hline $\begin{array}{l}\text { Instrumentation failure (screw fracture, lucency, } \\
\text { pullout) }\end{array}$ & 0 \\
\hline Overall survival rate, \% & 33.9 \\
\hline Death w/in 90 days & $9(13)$ \\
\hline $\begin{array}{l}\text { Median follow-up for those who survived }>90 \text { days, } \\
\text { mos }\end{array}$ & 25.3 \\
\hline
\end{tabular}

Values represent the number of patients (\%) unless stated otherwise.

Asymptomatic pulmonary cement embolism was detected in 1 patient $(1.45 \%)$ on postoperative CT. The patient was observed and did not develop any adverse sequelae to this finding or require anticoagulation.

During follow-up, 3 patients required additional spine surgery. One patient continued to have refractory axial back pain 2 years after surgery without CT evidence of instrumentation failure or lucency around the screws. The instrumentation was removed after lack of treatment response to palliative pain therapies. Two patients underwent additional surgery for extension of instrumentation for adjacent-level disease progression and cord compression. Follow-up CT imaging did not show any evidence of instrumentation failure, screw loosening, rod or screw fracture, or screw pullout.

\section{Discussion}

This study demonstrates the safety and efficacy of using cement-augmented fenestrated pedicle screws for cancer-related spinal instability. To our knowledge, this is the largest series describing the application of this technology in a tumor-specific patient population with descriptive analytics and clinical, radiographic, and patient-reported pain outcomes. The data presented show that when screws are well positioned within the pedicle and vertebral body without cortical breakthrough (Heary grade 1), the risk of cement leakage is low but still present. Moreover, our analysis shows that cement leakage most commonly occurs through the segmental veins, particularly when there is breach of the lateral pedicle or vertebral body, even with the tip of the screw and the fenestrations within the vertebral body. Extravascular spread outside the vertebral body was primarily associated with distal screw placement beyond the cortex (Heary grade 3 ), and cement extravasation into the spinal canal was primarily seen with medialized screws (Heary grade 4). Routine postoperative CT imaging in our study allowed for detailed analysis and characterization of cement extravasation.

With the use of intraoperative technological aids like computer-based navigation and robotics, improved accuracy of pedicle screw placement may arguably minimize the possibility of extravasation as seen with Heary grade 4 , which had the highest occurrence of cement in the basivertebral vein tracking into the spinal canal. When using fenestrated screws, careful planning of screw lengths and trajectories is required to avoid cortical wall disruption or having the fenestrations too close to the vertebral body wall, as weak bone may not contain the cement. In light of these observations, it is unclear whether the amount or type of extravasation has clinical significance. ${ }^{29}$

Although we observed cement extravasation with various screw positions, the number of breached screws is too small to establish well-defined associations among position, anatomical region, and cement extravasation, despite using published classification criteria. Screws that breach medially are angulated toward the basivertebral vein and may lead to cement leakage into the canal, but better understanding of the regional flow dynamics of the vertebral venous anatomy is needed to understand this better. Likewise, the vascularity associated with cancer infiltration into the bone may pose additional risk for extravasation and requires further quantitative study. In our study, the volume of cement injected was limited to $1.5 \mathrm{~mL}$ to control for potential operator-dependent variances that could account for extravasation. Similarly, consistent technique was applied to each case to minimize variance in cement preparation and delivery.

There are clearly other considerations that impact the pattern of cement fill that are dependent on more than just the placement of the screws, such as viscosity of the cement, the rate of injection, and underlying characteristics of the bone. Each of these have effects that are difficult to quantify. Developing better tools for assessing bone density prior to surgery may help select appropriate patients as well as plan which levels to augment with cement. In our series, $20 \%$ of patients were known to have osteoporosis and $40 \%$ had osteopenia, but it is not clear how knowing 
this before surgery would help mitigate cement extravasation. As an exercise, we measured the Hounsfield units (HU) for this cohort on preoperative CT scans, as very few had dual-energy x-ray absorptiometry scans, and we found that most of our patients had poor bone quality. However, further quantitative analysis was not possible given that CT-based HU osteopenia-osteoporotic cutoffs are not yet standardized or well defined and the heterogeneous tumor characteristics of the vertebrae make interpretation difficult. ${ }^{30-32}$ With more study, surrogate radiographic markers of bone density like HU or the recently published MRIbased vertebral bone quality score may help further associate the density of bone with fracture risk, instrumentation failure, and surgical outcomes. ${ }^{33-35}$

The safety of using cement in the spine is established, but complications are not uncommon..$^{20,36,37}$ Pulmonary embolism secondary to cement embolism has been reported in the surgical and interventional literature. ${ }^{38-42}$ Our finding is similar to other reports of asymptomatic cement embolus that did not cause hemodynamic or respiratory changes and did not require systemic anticoagulation. ${ }^{20}$ The need for additional surgery in our series was prompted by wound-related complications and adjacent-level metastatic disease progression. Alternative implants that do not require cement, such as expandable pedicle screws and hydroxyapatite-coated pedicle screws, may also improve fixation for poor bone quality, but further study is needed to assess their clinical efficacy in the metastatic spine setting. ${ }^{43,44}$

Regarding improvement in mechanical pain after surgery, this was demonstrated by changes in PROMIS scores for Pain Intensity and Pain Interference. This pain benefit is consistent with published data regarding the role of spine stabilization for mechanical instability. ${ }^{5}$ Barzilai et al. also demonstrated significant improvements in pain severity, intensity, and interference as measured with the Brief Pain Inventory at follow-up within 6 months after percutaneous stabilization with fenestrated screws. ${ }^{20}$ In 2004, the National Institutes of Health created PROMIS to improve patient self-reporting of pain, function, and quality of life. ${ }^{45}$ As a result, a wide range of these assessment tools are being increasingly used in degenerative, deformity, and oncological spine research. In the degenerative spine literature, these surveys have been shown to take less time to complete, have lower ceiling and floor effects, and perform as well as traditional outcomes surveys like the Oswestry Disability Index (ODI) and Neck Disability Index (NDI).

To date, only 2 studies have investigated PROMIS specifically for patient-reported pain outcomes for metastatic spine tumors. ${ }^{46,47}$ Paulino Pereira et al. ${ }^{46}$ compared the PROMIS Short Form 3a Pain Intensity with the Spine Oncology Study Group Outcome Questionnaire (SOSG-OQ) in a cohort of 46 surgical patients and found that PROMIS Pain Intensity was more reliable than the SOSG-OQ pain domain over a wider range of responses. The Pain Intensity survey is 3 items, asking the patient how intense their pain was in the last 7 days on average, at its worst, and at the present time. Similarly, Bernstein et al. studied a cohort of 36 patients who underwent surgery for spinal metastases and prospectively compared PROMIS Pain Interference with ODI and NDI and found strong correlations with the legacy surveys. ${ }^{47}$ The Pain Interference survey is 6 questions and assesses how pain interferes with social, cognitive, emotional, physical, and recreational activities over the last 7 days. Both studies suggest that PROMIS is advantageous to legacy outcomes instruments in assessing pain in patients with metastatic spinal tumors.

To our knowledge, the current study is the first to prospectively evaluate pain responses after surgery for spinal metastases using both PROMIS Pain Intensity and Pain Interference surveys. Prior to surgery, patients scored an average of 18.2 points above the normative population mean in Pain Interference and 26.9 points above the mean in Pain Intensity. These results highlight the burden of paindriven disabilities and to what extent pain can impact quality of life among patients with spinal metastases. Although patients experienced meaningful benefit after surgery, the average Pain Interference remained above the normative population mean, while the average Pain Intensity was below the mean, showing the disproportionate ways in which pain relief translates to daily life for these patients. These findings underscore the impact pain may have on the social, mental, emotional, and physical aspects of recovering from surgery. Although radiographic and stabilization goals can be seemingly satisfied with instrumentation systems like this, multidisciplinary advancements in pain management, rehabilitation, and palliative care are needed to help maximize the effectiveness of surgery for cancerrelated mechanical spine pain.

\section{Limitations}

This retrospective study analyzes a specific surgical treatment cohort without a comparative or control group. Clustering of data from a single tertiary referral institution may lead to unintended practice bias and may not necessarily be representative of other healthcare settings since geography affects the demographic and socioeconomic makeup of the studied population. Confounding from other unmeasured variables, such as the type of commercially available cement and differences in screw design across vendors, is also a limitation that this investigation was not powered to study. Utilization of fenestrated screw systems at our center was limited by their practical availability, and patients were not randomized to specific vendors or treatments. Best attempts were made to characterize the predominating cement pattern associated with each screw by discussion, but admittedly, any radiological interpretation is subject to a degree of variability. Further work is needed to critically analyze patient and surgical approach selection criteria (i.e., percutaneous vs open) as these technologies are integrated into practice. Prospective multicenter observational studies capturing varying construct types and their associated outcomes, complications, and impact on pain and quality of life are needed for comparative analysis. This study does not address or answer whether the additional time and cost related to these implants lead to reduced rates of instrumentation failure or superior pain benefit compared with conventional approaches.

\section{Conclusions}

Cement augmentation with fenestrated pedicle screws 
appears to be safe and effective for treating cancer-related instability in the spine. Although cement extravasation can occur with ideal screw placement, our findings show that this is generally asymptomatic, and the risk of thromboembolic events is minimal. Pain relief is consistent with established palliative benefits of stabilization for mechanical instability from cancer.

\section{References}

1. Rothrock RJ, Barzilai O, Reiner AS, et al. Survival trends after surgery for spinal metastatic tumors: 20 -year cancer center experience. Neurosurgery. 2021;88(2):402-412.

2. Hussain I, Barzilai O, Reiner AS, et al. Patient-reported outcomes after surgical stabilization of spinal tumors: symptombased validation of the Spinal Instability Neoplastic Score (SINS) and surgery. Spine J. 2018;18(2):261-267.

3. Newman WC, Laufer I, Bilsky MH. Neurologic, oncologic, mechanical, and systemic and other decision frameworks for spinal disease. Neurosurg Clin N Am. 2020;31(2):151-166.

4. Roodman GD. Mechanisms of bone metastasis. $N$ Engl $J$ Med. 2004;350(16):1655-1664.

5. Hussain I, Barzilai O, Reiner AS, et al. Spinal Instability Neoplastic Score component validation using patient-reported outcomes. J Neurosurg Spine. 2019;30(4):432-438.

6. Amankulor NM, Xu R, Iorgulescu JB, et al. The incidence and patterns of hardware failure after separation surgery in patients with spinal metastatic tumors. Spine J. 2014;14(9):1850-1859.

7. Pedreira R, Abu-Bonsrah N, Karim Ahmed A, et al. Hardware failure in patients with metastatic cancer to the spine. $J$ Clin Neurosci. 2017;45:166-171.

8. Longo M, De la Garza Ramos R, Gelfand Y, et al. Incidence and predictors of hardware failure after instrumentation for spine metastasis: a single-institutional series. World Neurosurg. 2019;125:e1170-e1175.

9. Mesfin A, Sciubba DM, Dea N, et al. Changing the adverse event profile in metastatic spine surgery: an evidence-based approach to target wound complications and instrumentation failure. Spine (Phila Pa 1976). 2016;41(suppl 20):S262-S270.

10. Schilling AT, Ehresman J, Huq S, et al. Risk factors for wound-related complications after surgery for primary and metastatic spine tumors: a systematic review and meta-analysis. World Neurosurg. 2020;141:467-478.e3.

11. Lau D, Leach MR, Than KD, et al. Independent predictors of complication following surgery for spinal metastasis. Eur Spine J. 2013;22(6):1402-1407.

12. Massaad E, Fatima N, Hadzipasic M, et al. Predictive analytics in spine oncology research: first steps, limitations, and future directions. Neurospine. 2019;16(4):669-677.

13. Elder BD, Ishida W, Goodwin CR, et al. Bone graft options for spinal fusion following resection of spinal column tumors: systematic review and meta-analysis. Neurosurg Focus. 2017;42(1):E16.

14. Molina C, Goodwin CR, Abu-Bonsrah N, et al. Posterior approaches for symptomatic metastatic spinal cord compression. Neurosurg Focus. 2016;41(2):E11.

15. Hofstetter CP, Chou D, Newman CB, et al. Posterior approach for thoracolumbar corpectomies with expandable cage placement and circumferential arthrodesis: a multicenter case series of 67 patients. J Neurosurg Spine. 2011;14(3):388-397.

16. Frankel BM, Jones T, Wang C. Segmental polymethylmethacrylate-augmented pedicle screw fixation in patients with bone softening caused by osteoporosis and metastatic tumor involvement: a clinical evaluation. Neurosurgery. 2007;61(3):531-538.

17. Moussazadeh N, Rubin DG, McLaughlin L, et al. Shortsegment percutaneous pedicle screw fixation with cement augmentation for tumor-induced spinal instability. Spine $J$. 2015;15(7):1609-1617.
18. Elder BD, Lo SF, Holmes C, et al. The biomechanics of pedicle screw augmentation with cement. Spine J. 2015;15(6): 1432-1445.

19. Frankel BM, D'Agostino S, Wang C. A biomechanical cadaveric analysis of polymethylmethacrylate-augmented pedicle screw fixation. J Neurosurg Spine. 2007;7(1):47-53.

20. Barzilai O, McLaughlin L, Lis E, et al. Utility of cement augmentation via percutaneous fenestrated pedicle screws for stabilization of cancer-related spinal instability. Oper Neurosurg (Hagerstown). 2019;16(5):593-599.

21. Fisher CG, DiPaola CP, Ryken TC, et al. A novel classification system for spinal instability in neoplastic disease: an evidence-based approach and expert consensus from the Spine Oncology Study Group. Spine (Phila Pa 1976). 2010; 35(22):E1221-E1229.

22. Massaad E, Hadzipasic M, Alvarez-Breckenridge C, et al. Predicting tumor-specific survival in patients with spinal metastatic renal cell carcinoma: which scoring system is most accurate? J Neurosurg Spine. 2020;33(4):529-539.

23. Amtmann D, Cook KF, Jensen MP, et al. Development of a PROMIS item bank to measure pain interference. Pain. 2010; 150(1):173-182.

24. Cook KF, Dunn W, Griffith JW, et al. Pain assessment using the NIH Toolbox. Neurology. 2013;80(11)(suppl 3):S49-S53.

25. Laufer I, Iorgulescu JB, Chapman T, et al. Local disease control for spinal metastases following "separation surgery" and adjuvant hypofractionated or high-dose single-fraction stereotactic radiosurgery: outcome analysis in 186 patients. $J$ Neurosurg Spine. 2013;18(3):207-214.

26. Heary RF, Bono CM, Black M. Thoracic pedicle screws: postoperative computerized tomography scanning assessment. J Neurosurg. 2004;100(4 Suppl Spine):325-331.

27. Gertzbein SD, Robbins SE. Accuracy of pedicular screw placement in vivo. Spine (Phila Pa 1976). 1990;15(1):11-14.

28. Yeom JS, Kim WJ, Choy WS, et al. Leakage of cement in percutaneous transpedicular vertebroplasty for painful osteoporotic compression fractures. J Bone Joint Surg Br. 2003; 85(1):83-89.

29. Lador R, Dreiangel N, Ben-Galim PJ, Hipp JA. A pictorial classification atlas of cement extravasation with vertebral augmentation. Spine J. 2010;10(12):1118-1127.

30. Wang P, She W, Mao Z, et al. Use of routine computed tomography scans for detecting osteoporosis in thoracolumbar vertebral bodies. Skeletal Radiol. 2021;50(2):371-379.

31. Booz C, Noeske J, Albrecht MH, et al. Diagnostic accuracy of quantitative dual-energy CT-based bone mineral density assessment in comparison to Hounsfield unit measurements using dual x-ray absorptiometry as standard of reference. Eur J Radiol. 2020;132:109321.

32. Zaidi Q, Danisa OA, Cheng W. Measurement techniques and utility of Hounsfield unit values for assessment of bone quality prior to spinal instrumentation: a review of current literature. Spine (Phila Pa 1976). 2019;44(4):E239-E244.

33. Ehresman J, Schilling A, Yang X, et al. Vertebral bone quality score predicts fragility fractures independently of bone mineral density. Spine J. 2021;21(1):20-27.

34. Bredow J, Boese CK, Werner CM, et al. Predictive validity of preoperative CT scans and the risk of pedicle screw loosening in spinal surgery. Arch Orthop Trauma Surg. 2016;136(8): 1063-1067.

35. Schreiber JJ, Anderson PA, Hsu WK. Use of computed tomography for assessing bone mineral density. Neurosurg Focus. 2014;37(1):E4.

36. Berenson J, Pflugmacher R, Jarzem P, et al. Balloon kyphoplasty versus non-surgical fracture management for treatment of painful vertebral body compression fractures in patients with cancer: a multicentre, randomised controlled trial. Lancet Oncol. 2011;12(3):225-235.

37. Fourney DR, Schomer DF, Nader R, et al. Percutaneous verte- 
broplasty and kyphoplasty for painful vertebral body fractures in cancer patients. J Neurosurg. 2003;98(1)(suppl):21-30.

38. Ulusoy OL, Kahraman S, Karalok I, et al. Pulmonary cement embolism following cement-augmented fenestrated pedicle screw fixation in adult spinal deformity patients with severe osteoporosis (analysis of 2978 fenestrated screws). Eur Spine J. 2018;27(9):2348-2356.

39. Martín-Fernández M, López-Herradón A, Piñera AR, et al. Potential risks of using cement-augmented screws for spinal fusion in patients with low bone quality. Spine J. 2017;17(8): 1192-1199.

40. Janssen I, Ryang YM, Gempt J, et al. Risk of cement leakage and pulmonary embolism by bone cement-augmented pedicle screw fixation of the thoracolumbar spine. Spine J. 2017; 17(6):837-844.

41. Wang LJ, Yang HL, Shi YX, et al. Pulmonary cement embolism associated with percutaneous vertebroplasty or kyphoplasty: a systematic review. Orthop Surg. 2012;4(3):182-189.

42. Krueger A, Bliemel C, Zettl R, Ruchholtz S. Management of pulmonary cement embolism after percutaneous vertebroplasty and kyphoplasty: a systematic review of the literature. Eur Spine J. 2009;18(9):1257-1265.

43. Tandon V, Franke J, Kalidindi KKV. Advancements in osteoporotic spine fixation. J Clin Orthop Trauma. 2020;11(5): 778-785.

44. Ohe M, Moridaira H, Inami S, et al. Pedicle screws with a thin hydroxyapatite coating for improving fixation at the bone-implant interface in the osteoporotic spine: experimental study in a porcine model. J Neurosurg Spine. 2018;28(6):679-687.

45. Cella D, Yount S, Rothrock N, et al. The Patient-Reported Outcomes Measurement Information System (PROMIS): progress of an NIH Roadmap cooperative group during its first two years. Med Care. 2007;45(5)(suppl 1):S3-S11.

46. Paulino Pereira NR, Janssen SJ, Raskin KA, et al. Most efficient questionnaires to measure quality of life, physical function, and pain in patients with metastatic spine disease: a cross-sectional prospective survey study. Spine J. 2017;17(7): 953-961.
47. Bernstein DN, Bakhsh W, Papuga MO, et al. An evaluation of PROMIS in patients with primary or metastatic spine tumors. Spine (Phila Pa 1976). 2019;44(10):747-752.

\section{Disclosures}

The authors report no conflict of interest concerning the materials or methods used in this study or the findings specified in this paper.

\section{Author Contributions}

Conception and design: Shin, Massaad. Acquisition of data: Shin, Massaad, Rolle. Analysis and interpretation of data: Shin, Massaad, Rolle. Drafting the article: Shin, Massaad. Critically revising the article: all authors. Reviewed submitted version of manuscript: all authors. Approved the final version of the manuscript on behalf of all authors: Shin. Statistical analysis: Shin, Massaad. Administrative/technical/material support: Shin, Massaad. Study supervision: Shin.

\section{Supplemental Information \\ Previous Presentations}

Portions of this work were presented as a Best Abstract oral presentation at Spine Summit 2018-Annual Meeting of the AANS/ CNS Section on Disorders of the Spine and Peripheral Nerves, Orlando, Florida, March 14-17, 2018.

\section{Correspondence}

John H. Shin: Massachusetts General Hospital, Boston, MA. shin.john@mgh.harvard.edu. 